\title{
The influence of mortality and socioeconomic status on risk and delayed rewards: A replication with British participants
}

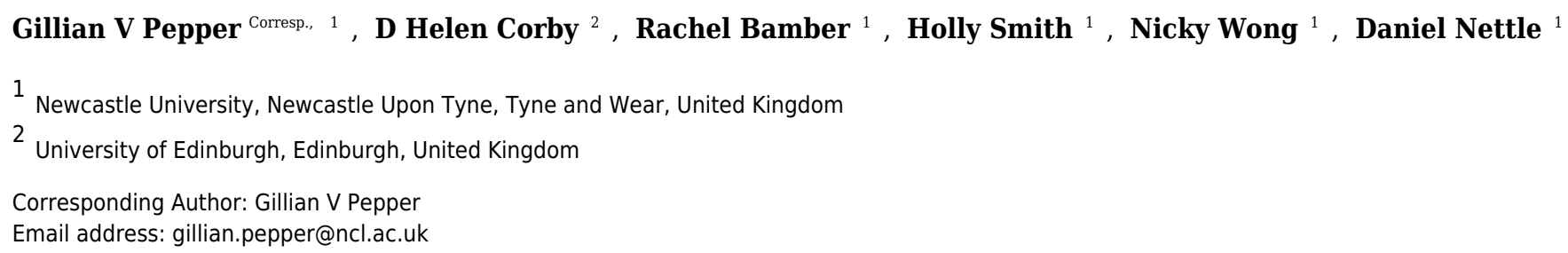

Here, we report three attempts to replicate a finding from an influential psychological study [Griskevicius, V. et al. J. Pers. Soc. Psych. 100: 1015-26, 2011]. The original study found interactions between childhood SES and experimental mortality-priming condition in predicting risk acceptance and delay discounting outcomes. The original study used US student samples. We used British university students (replication 1) and British online samples (replications 2 and 3 ) with a modified version of the original priming material, which was tailored to make it more credible to a British audience. We did not replicate the interaction between childhood SES and mortality-priming condition in any of our three experiments. The only consistent trend of note was an interaction between sex and priming condition for delay discounting. We note that psychological priming effects are considered fragile and often fail to replicate. Our failure to replicate the original finding could be due to demographic differences in study participants, alterations made to the prime, or other study limitations. However, it is also possible that the previously reported interaction is not a robust or generalizable finding. 


\section{PeerJ}

\section{Manuscript to be reviewed}

1 Title: The influence of mortality and socioeconomic status on risk and delayed rewards: A

2 replication with British participants

3 Authors: Gillian V. Pepper ${ }^{1}$, D. Helen Corby $^{2}$, Rachel Bamber ${ }^{1}$, Holly Smith ${ }^{1}$, Nicky Wong ${ }^{1}$, 4 \& Daniel Nettle ${ }^{1}$

5 Affiliations: 1. Newcastle University, UK

6

2. The University of Edinburgh, UK

7 Corresponding author: Gillian V. Pepper, gillian.pepper@ncl.a.uk 


\section{Manuscript to be reviewed}

\section{Abstract}

10 Here, we report three attempts to replicate a finding from an influential psychological study

11 [Griskevicius, V. et al. J. Pers. Soc. Psych. 100: 1015-26, 2011]. The original study found

12 interactions between childhood SES and experimental mortality-priming condition in

13 predicting risk acceptance and delay discounting outcomes. The original study used US

14 student samples. We used British university students (replication 1) and British online

15 samples (replications 2 and 3) with a modified version of the original priming material, which

16 was tailored to make it more credible to a British audience. We did not replicate the

17 interaction between childhood SES and mortality-priming condition in any of our three

18 experiments. The only consistent trend of note was an interaction between sex and priming

19 condition for delay discounting. We note that psychological priming effects are considered

20 fragile and often fail to replicate. Our failure to replicate the original finding could be due to

21 demographic differences in study participants, alterations made to the prime, or other study

22 limitations. However, it is also possible that the previously reported interaction is not a robust

23 or generalizable finding.

\section{Background}

In recent years scientists in fields ranging from biomedicine to psychology have become increasingly concerned with the difficulties of replicating findings that are often assumed to be universal and reproducible (Cesario, 2014; Ferguson \& Mann, 2014; Moonesinghe, Khoury, \& Janssens, 2007). In particular, experimental psychologists have been concerned about the fragility of priming effects, highlighting the need for replication of priming experiments (Cesario, 2014; Moonesinghe et al., 2007). In this paper, we report our attempts to replicate a finding of particular interest to us, which has been influential and highly cited.

The study we sought to replicate was that of Griskevicius, Tybur, Delton, and Robertson (2011). Across three experiments, they found that, following exposure to a mortality-risk prime (a fake newspaper article about rising violent crime, designed to elicit the sense that the world is dangerous and unpredictable), participants who grew up wealthier took fewer risks in a lab-based risky choice task, while those who grew up poorer took more risks. The same mortality-risk prime led participants who grew up wealthier to prefer future rewards to immediate ones in a lab-based delay discounting task. Meanwhile, those who grew up in poorer environments preferred more immediate rewards after mortality priming. Thus, for both risk acceptance and delay discounting, there were interactions between priming condition and childhood socioeconomic status in predicting the outcome. Only the interactions were significant in the original study: there were no overall directional effects of either the prime, or childhood socioeconomic status. This finding of an interaction between acute mortality priming and childhood socioeconomic background has been widely cited. However, there have been no direct replications of the original experiments. The original study used student participants from a large university in the U.S.A. Here, we report three replications using British samples, one sample of university students, and two online samples. As the three experiments reported in the original paper were extremely similar to one another,

49 both in design and in outcome, we focussed on a single one (experiment 2), performing 50 multiple replications to maximize the precision of our estimates of the effects. We chose to 51 focus on replicating experiment 2 because the control condition did not use a prime. Using 
52 only one adapted prime, rather than two, meant that our replication was closer to the original 53 study.

\section{Methods}

55 We carried out three experiments that replicated experiment 2 from Griskevicius et al. 56 (2011). These studies were granted ethical approval by the Newcastle University Faculty of 57 Medical Sciences ethics committee (reference number 00554). The pre-registered protocols 58 are available online at https://osf.io/6ucmq/ and https://osf.io/drq98/. All aspects of the 59 replications including the informed consent and debrief screens, demographic questions, 60 prime presentation, and outcome measures (see Measures), were presented in a web browser 61 via Qualtrics (Qualtrics, Provo, UT, C2016). Participants in the lab-based study (replication 62 1) received an additional verbal debrief.

63 As in Griskevicius et al (2011), participants in all three experiments were told that the 64 newspaper article they read in the mortality priming condition (see Priming material) was 65 part of a memory test. They were told that, after reading the article, they would complete 66 questionnaires related to financial preferences to allow for memory decay. Participants were 67 randomly allocated to either the mortality-priming condition or the control condition by 68 Qualtrics. In the control condition, there were no priming materials. Control participants 69 simply indicated their preferences for the risky and delayed rewards (see Measures). The order of presentation for the risky choice and delay discounting outcome measures was randomised in all conditions. Following this, participants in all conditions indicated their childhood socioeconomic status (SES) by answering the questions outlined below (see Measures).

\section{Participants and recruitment}

The experimental treatments and the variables collected (see Measures) were the same in all three of our experiments. The key differences between the three experiments were in the participant demographics, and the method of recruitment: For our first replication, which took place in a laboratory at Newcastle University, the participants were 72 undergraduate University students (mean age $=20,9$ male, 63 female, see Table 1). The sample size for this first replication aimed to match that of the original experiment, which had 71 participants. For our second replication, 159 participants (mean age $=39,85$ male, 74 female, Table 1) were recruited online via Crowdflower (http://www.crowdflower.com). Crowdflower is an internet crowdsourcing platform through which users can be paid to complete online tasks, including surveys and experiments (for a useful review of Crowdsourcing platforms as research tools, see Peer, Brandimarte, Samat, \& Acquisti, 2017). For our third replication, 162 participants (mean age $=36,85$ male, 77 female, Table 1) were recruited via Crowdflower and, if allocated to the priming condition, were asked additional questions about the prime as a manipulation check (see sections on Priming material and The effects of prime perception). In both online experiments (replications $2 \& 3$ ), 200 participants were initially recruited. Responses from those participants were then subjected to a series of quality-control checks. The quality control checks were designed to ensure that participants were really from the UK, that they were not repeat participants, and to increase the likelihood that they had been adequately exposed to the prime. After these checks, we were left with 159

94 participants in replication 2, and 162 participants in replication 3 (Table 1). 
95 The quality checks used were as follows: 1) that participant identification numbers matched

96 on Qualtrics and Crowdflower and were unique (if participants had attempted to complete the study multiple times, only the data from their first attempts were included in our analyses); 2) that the participants took a reasonable time (established using timestamp data from our labbased replication) to complete the study: data were excluded from participants who took less than 60 seconds (the minimum time needed to honestly complete the experiment in the control condition), or more than 15 minutes (a long completion time indicates that a participant may have been interrupted during the experiment, potentially allowing any priming effect to wear off); 3) that the participants completed the experiment from a device with a UK-based internet protocol (IP) address (our adapted priming material described violent crimes in the UK and so might not have been effective for participants living in other countries), and that the IP addresses matched between the Crowdflower and Qualtrics platforms (a further verification that the location information was genuine); 4) that participants declared themselves to be current UK residents when explicitly asked; 5) that the participants had entered the correct verification code (generated by Qualtrics at the end of the experiment) via the Crowdflower platform.

\section{Priming material}

112 The original experiment ( 2 of 3 by Griskevicius et al., 2011), used two conditions - one 113 mortality prime, and one control condition in which there was no prime. The original 114 mortality prime was a fake New York Times story entitled "Dangerous Times Ahead: Life and Death in the 21st Century," describing violent trends in the U.S.A. We adapted this

116 newspaper story (provided by Griskevicius et al., personal communication) for a British 117 audience, altering descriptions of shootings so that they were about stabbings (which are 118 more plausible events in the UK) and mentioning terrorist attacks that had occurred in the 119 UK, rather than in the U.S.A. In doing so, we deleted 108 words from the original prime, and 120 added 131 words. The adapted prime is available as part of our pre-registered protocol, which 121 can be seen online at https://osf.io/6ucmq/.

122 We pilot tested the adapted prime with a sample of 23 British students ( 7 male, 16 female) in 123 order to ensure that the adapted article had similar effects to the original. Our pilot participants came from a range of socioeconomic backgrounds, as measured by the Index of Multiple deprivation (IMD) ranks for their home postcodes, which ranged from 217 to 31,805 (from a possible range of $1-32,844$ ). The IMD identifies deprived areas of the country by combining a range of economic and social indicators into a single score. These scores are considered a meaningful objective measure of socioeconomic status (Danesh, Gault,

129 Semmence, Appleby, \& Peto, 1999; McLennan, Barnes, Noble, Davies, \& Garratt, 2011).

130 Griskevicius, Delton, Robertson, and Tybur (2011) originally tested their prime for its effect 131 on perceptions of safety, unpredictability and general arousal. We piloted our prime using the same questions, which were as follows: 1) "To what extent did the story make you think the world will become a more dangerous place?" 2) "To what extent did the story make you think

134 the world will become unsafe?" 3) "To what extent did the story make you think the world 135 will become more unpredictable?" 4) "To what extent did the story make you think the world 136 will become uncertain?" 5) "To what extent did the story make you feel more emotionally 137 aroused?" In addition to these questions, we added a question about how convincing our 138 raters thought the article was: "Did you find this article convincing?" All of these prime 
139 piloting questions were answered on a 7-point likert scale, with 1 being "not at all" and 7

140 being "very much".

141 We found that our prime had a similar effect on general arousal and perceptions of danger to 142 those found in the original prime piloting by Griskevicius et al., (2011). However, it had 143 significantly less of an effect on perceptions of uncertainty (Table 2). Pilot participants found 144 the article moderately convincing, the mean rating being 4.04 out of a possible 7 .

\section{Measures}

146 Participants in all three experiments were asked for their age, sex, and home postcode. Their 147 postcodes were used to obtain Index of Multiple Deprivation (IMD) scores, which are 148 considered a good measure of socioeconomic status (SES) for people living in the UK 149 (Danesh et al., 1999). This measure was not used by Griskevicius et al. (2011), but provided 150 us with an objective measure of socioeconomic status, to be used alongside subjective 151 childhood socioeconomic status (see Supplementary Materials). As in the original study, 152 childhood SES was measured by asking participants to rate their agreement (on a scale from 1531 to 7) with the following statements: (a) "My family usually had enough money for things 154 when I was growing up"; (b) "I grew up in a relatively wealthy neighbourhood"; (c) "I felt 155 relatively wealthy compared to the other kids in my school." Agreement with these 156 statements was then summed to create the childhood SES score. Subjective adult 157 socioeconomic status was measured by asking participants to rate their agreement (on a scale 158 from 1 to 7) with the following statements: (a) "I have enough money to buy things I want"; 159 (b) "I don't need to worry too much about paying my bills"; (c) "I don't think I'll have to 160 worry about money too much in the future." Again, participants' agreement with these 161 statements was summed to create an adult SES score. The associations between these 162 subjective scores and postcode-based deprivation scores for each replication are reported in 163 the Supplementary Materials (Table S4).

164 We used the same outcome measures as the original study (Griskevicius, Tybur, et al., 2011). 165 After having read the fake newspaper article (mortality-priming condition), or not (control 166 condition), participants answered questions designed to measure risk preferences or delay 167 discounting (in randomised order). The risk preference questions were seven choices of the 168 format, "Do you want a 50\% chance of getting $\$ 800$ OR £__for sure?" with the certain 169 amount increasing from $£ 100$ to $£ 700$ in $£ 100$ increments. For delay discounting, participants 170 were offered seven choices structured as follows, "Do you want to get 1100 tomorrow OR $171 £ \quad 90$ days from now?" with the delayed reward starting at $£ 110$ and increasing to $£ 170$ in $172 £ 10$ increments. As in the original study, a higher score on this measure indicates greater 173 patience (less-steep discounting). For fuller details, see our protocol at https://osf.io/6ucmq/.

\section{Analysis}

175 Analyses were carried out in R 3.1.3 using the ggplot (Wickham, 2009), dplyr (Wickham, 176 2016), car (Fox, 2016), psych (Revelle, 2016), metaphor (Viechtbauer, 2010), and pwr 177 (Champely, 2017) packages. The data and $\mathrm{R}$ scripts used for the analyses are available as 178 Supplementary Materials. The main analyses reported in this paper were general linear 179 models including as predictors: the main effect of mortality-priming condition (prime or no 180 prime); the main effect of childhood SES; and the interaction between mortality-priming 181 condition and childhood SES. In addition, following Griskevicius et al. (2011), we performed 
182 a number of additional exploratory analyses in which adult SES and sex were added as

183 additional predictors and 3-way interactions were explored. These extra analyses are reported

184 in the Supplementary Materials (section 3). We report standard two-tailed significance tests.

185 However, following the original analysis by Griskevicius et al. (2011) we also calculated

186 directed $p$-values $\left(\mathrm{p}_{\text {dir }}\right)$ for the critical interaction between condition and childhood SES.

187 Directed tests are intended to enhance power relative to two-tailed significance tests, without

188 ignoring the possibility of effects in the unexpected direction (Rice \& Gaines, 1994).

189 Having completed our three replications, we meta-analysed them, using a random-effects 190 meta-analysis model implemented in the 'metafor' package (Viechtbauer, 2010). This was to 191 investigate the possibility that there might be small effects, not significant in any one of the 192 replications considered individually, but detectable when the information from all three 193 replications was combined.

194 At the end of our third replication, after the outcome measures had been recorded, we 195 presented participants with the questions initially used for prime piloting, as a manipulation 196 check (see Priming material). We used general linear models to examine whether 197 participants' responses to the primes predicted their risk or delay discounting responses in the 198 priming condition, and whether they did so in interaction with childhood SES.

199 Results

\section{$200 \quad$ Individual replications}

201 The results of the general linear models for our three replications are summarised in table 3.

202 The critical interaction was not significant in any of the experiments, either by two-tailed p203 values or directed tests. The main effects were largely non-significant. Figures 1, 2 and 3 204 reproduce for our three replications the plots used by Griskevicius et al. (2011) to illustrate 205 the interactions in their data.

\section{The effects of prime perception}

207 To address the possibilities that we did not replicate the original findings in our first two 208 attempts either because we had altered the prime, or because the prime was less credible to 209 British participants, we collected additional data during replication 3: After the outcome 210 variables had been recorded, participants in the mortality-priming condition were also 211 presented with the prime-piloting questions, described under Priming material.

212 We investigated whether participants' belief in the primes predicted their risk acceptance or 213 delay discounting scores. In models controlling for childhood SES, the extent to which 214 participants reported finding the prime convincing had no effect on their risk or delay 215 discounting scores (risk $\mathrm{F}_{1,83}=0.17, \mathrm{p}=0.68$; discounting $\mathrm{F}_{1,83}=2.08, \mathrm{p}=0.15$ ). However, 216 exploratory analyses revealed that participants who felt that the world was unsafe after 217 reading the prime were also subsequently less willing to accept risky options $\left(\mathrm{F}_{1,79}=6.37, \mathrm{p}\right.$ $218=0.01)$. There was also a trend in which participants who reported feeling that the world 219 would become more dangerous after reading the prime also discounted future rewards less steeply $\left(\mathrm{F}_{1,79}=3.23, \mathrm{p}=0.07\right)$. Thus, we ran models testing for interactions between childhood SES and post-prime perceptions that the world was unsafe or dangerous - to test whether interaction effects with childhood SES would be visible in those participants that had been more-successfully primed with a sense of danger. The main effects of primed threat 
224 perceptions on risk and delay discounting remained significant in these models. However,

225 there were no interactions between childhood SES and primed perceptions for risk and delay

226 discounting (Table 4).

\section{Meta-analysis}

228 When we meta-analysed the findings of the three experiments, the $95 \%$ confidence intervals 229 for the parameter estimates overlapped zero in most cases, for both main effects and 230 interactions (figure 4). Thus, we did not detect interaction effects in the individual 231 replications, or when all three replications were combined.

\section{Additional analyses}

233 In the additional analyses reported in the Supplementary Materials, the only recurrent finding

234 was a trend towards an interaction between sex and mortality-priming condition in predicting 235 delay discounting. This interaction was marginally non-significant in each individual 236 replication, but was significant in a meta-analysis across all three replications $(\mathrm{B}=1.66$, 237 s.e.(B) $=0.55, \mathrm{z}=3.00, \mathrm{p}<0.01$ ). After priming, men became more patient (that is, 238 discounted the future less steeply), whereas women became less patient (that is, discounted 239 more steeply; see Supplementary Materials, figures S1 and S2).

\section{Discussion}

241 We have reported three attempts to replicate findings by Griskevicius et al., (2011), who 242 found consistent interaction effects between childhood SES and mortality-priming condition 243 for two outcomes: risk acceptance and delay discounting. We did not replicate this main 244 finding in our three experiments which, unlike the original study, used British participants. In none of our individual replications was the predicted interaction significantly different from zero. We found no evidence of an interaction when we combined the results of the three replications in a meta-analysis. Our samples were diverse (one student, two online), and contained a reasonable degree of variation in childhood SES, as would have been necessary to reveal an interaction. We cannot directly compare the childhood SES variation in our sample with that in the sample of Griskevicius et al. (2011), as they do not report descriptive statistics, but it seems unlikely our samples contained much less variation.

252 There are a number of potential reasons for our failure to replicate the original findings of 253 Griskevicius et al., (2011). Firstly, we used British undergraduate students (replication 1) and online participants from the more general British population (replications $2 \& 3$ ), whilst the original study used a North American undergraduate sample. Thus, demographic and cultural differences could explain differences in either our participants' behavioural responses to the priming material, or their willingness to believe the information given in the prime. Indeed, we altered the original priming material in order to make it more convincing to a British audience (for example by replacing references to gun violence with references to knife attacks - see Priming material). Using the same piloting questions as Griskevicius et al., we found that our prime had similar effects on perceived danger (dangerous, unsafe: Table 2) in its respective audience to the original prime. However, our prime had significantly less effect on perceived uncertainty (uncertain, unpredictable: Table 2). In addition, we asked piloting participants how convincing they found the priming article. In both the initial pilot (Table 2), and in replication 3 (supplement, Table S13), participants were only moderately convinced by the priming information (4-5 points on a 7-point scale, with a score of 7 signifying that the 
267 prime was very convincing). Griskevicius et al. (2011) did not ask their pilot participants to

268 rate the prime for convincingness, and so we are unable to directly compare, but it is possible

269 that our participants found our version of the prime less convincing than participants in the

270 original experiments.

271 Our replications were also limited in other ways. Although our first replication matched the

272 original study sample size $\left(\mathrm{n}_{1}=72\right.$, Table 1$)$, it may well have been underpowered. Indeed,

273 power analyses indicated that, assuming $80 \%$ power and a significance level of 0.05 ,

274 replication 1 was powered to detect a minimum detectable main effect (MDE) of 0.11 . This is

275 a small-to-medium effect according to convention (Champely, 2017). Replications 2 and 3

276 had much greater power $\left(\mathrm{n}_{2}=159, \mathrm{MDE}_{2}=0.05, \mathrm{n}_{3}=162, \mathrm{MDE}_{3}=0.05\right)$, but used an online

277 crowdsourcing platform for participant recruitment. We applied rigorous quality controls (see

278 Participants and recruitment) to ensure that the data came from participants living in the UK

279 (the target audience for the modified prime), and that the participants didn't take too long (an

280 indicator that priming may have been interrupted) or too short (an indicator that the

281 participants may have answered questions without reading them thoroughly) a time to

282 complete the study. Nonetheless, we had no control over the environments in which the

283 online participants experienced the experiment-something which may have affected the

284 efficacy of priming. Finally, crowdsourcing platforms have limited systems in place to

285 prevent their users "cheating" (e.g. by taking part in a study twice, Peer et al., 2017). We tried

286 to reduce this problem by excluding repeat attempts from the same IP address (see

287 Participants and recruitment). However, this may not be sufficient to prevent a few users

288 repeatedly participating from different devices.

289 Across our three experiments, we saw some evidence that participants from higher-childhood

290 SES backgrounds were more accepting of risk. This result was marginally non-significant

291 overall. However, the associations were stronger in the two non-student samples, where the

292 variation in childhood SES was larger. We also found, for delay discounting, an interaction

293 between sex and priming that was significant across the three studies in meta-analysis: men

294 tended to respond to the prime by becoming more patient, whereas women tended to become

295 less patient (see supplement Figures S1 \& S2). As this effect was not the subject of an a priori

296 prediction, and was not reported by Griskevicius et al. (2011), we interpret it with caution.

297 We found no evidence of a three-way interaction between prime, sex, and childhood SES (see

298 supplement, section 3), and thus there is no reason to believe that differing sex balances in

299 our samples and those of Griskevicius et al. (2011) explain this difference in results.

300 Although it is possible that cross-cultural, demographic or methodological differences might

301 account for our failure to replicate the original finding, it is also possible that the original

302 findings were a false positive. Set against this possibility is that fact that Griskevicius et al.

303 (2011) reported three similar experiments using the same materials, and obtained the same

304 result each time. We note that one attempt at conceptually replicating the effect in US

305 undergraduate students also found no evidence of the interaction (Frederick, Khan, \&

306 Ancona, 2016). However, this study by Frederick et al. used different priming material and,

307 as the authors note, priming effects seem to be particularly sensitive to methodological

308 differences (Cesario, 2014). Thus we suggest that further attempts at replication, both in the

309 US population and in other populations internationally, are needed.

\section{Acknowledgements}




\section{PeerJ}

Manuscript to be reviewed

311 We are grateful to our participants for providing data, and to Katherine Corker and one other

312 anonymous reviewer for their helpful comments and suggestions.




\section{Manuscript to be reviewed}

\section{References}

315 Cesario, J. (2014). Priming, Replication, and the Hardest Science. Perspectives on Psychological Science, 9(1), 40-48. http://doi.org/10.1177/1745691613513470

317 Champely, S. (2017). pwr: Basic Functions for Power Analysis.

Danesh, J., Gault, S., Semmence, J., Appleby, P., \& Peto, R. (1999). Postcodes as useful markers of social class: population based study in 26000 British households Commentary: Socioeconomic position should be measured accurately. BMJ, 318(7187), 843-845. http://doi.org/10.1136/bmj.318.7187.843

Ferguson, M. J., \& Mann, T. C. (2014). Effects of evaluation: An example of robust "social" priming. Social, 32, 33-46.

Fox, J. (2016). car: Companion to Applied Regression.

325

326

327

328

329

330

331

332

333

334

335

336

337

338

339

340

341

342

343

344

345

346

347

348

Frederick, M., Khan, H., \& Ancona, M. (2016). Does the thought of death accelerate a fast life history strategy?: Evaluating a mortality salience prime. EvoS Journal: The Journal of the Evolutionary Studies Consortium, NEEPS Spec(September), 13-21.

Griskevicius, V., Delton, A. W., Robertson, T. E., \& Tybur, J. M. (2011). Environmental contingency in life history strategies: the influence of mortality and socioeconomic status on reproductive timing. Journal of Personality and Social Psychology, 100(2), 241-54. http://doi.org/10.1037/a0021082

Griskevicius, V., Tybur, J. M., Delton, A. W., \& Robertson, T. E. (2011). The influence of mortality and socioeconomic status on risk and delayed rewards: A life history theory approach. Journal of Personality and Social Psychology, 100(6), 1015-26. http://doi.org/10.1037/a0022403

McLennan, D., Barnes, H., Noble, M., Davies, J., \& Garratt, E. (2011). The English Indices of Deprivation 2010 - technical report. Social Policy. Department for Communities and Local Government.

Moonesinghe, R., Khoury, M. J., \& Janssens, A. C. J. W. (2007). Most published research findings are false-but a little replication goes a long way. PLoS Medicine, 4(2), e28. http://doi.org/10.1371/journal.pmed.0040028

Peer, E., Brandimarte, L., Samat, S., \& Acquisti, A. (2017). Journal of Experimental Social Psychology Beyond the Turk: Alternative platforms for crowdsourcing behavioral research. Journal of Experimental Social Psychology, 70, 153-163. http://doi.org/10.1016/j.jesp.2017.01.006

Revelle, W. (2016). psych: Procedures for Personality and Psychological Research. Northwestern University, Evanston, Illinois, USA.

Rice, W. R., \& Gaines, S. D. (1994). "Heads I win, tails you lose": Testing directional alternative hypotheses in ecological and evolutionary research. Trends in Ecology and Evolution, 9(6), 235-237. http://doi.org/10.1016/0169-5347(94)90258-5

Viechtbauer, W. (2010). Conducting Meta-Analyses in R with the metafor Package. Journal of Statistical Software, 36(3), 1-48. http://doi.org/10.1103/PhysRevB.91.121108

Wickham, H. (2009). ggplot2: Elegant Graphics for Data Analysis. Springer-Verlag New 
York.

355 Wickham, H. (2016). dplyr: A Grammar of Data Manipulation.

356

\begin{tabular}{|c|c|c|c|c|}
\hline & $\begin{array}{c}\text { Original } \\
\text { experiment }\end{array}$ & UK replication 1 & UK replication 2 & UK replication 3 \\
\hline $\mathrm{n}$ & 71 & 72 & 159 & 162 \\
\hline Males, females & 36,35 & 9,63 & 85,74 & 85,77 \\
\hline Mean age (sd) & $20.8(\mathrm{nr})$ & $19.8(2.0)$ & $38.9(11.5)$ & $36.3(11.9)$ \\
\hline Mean child SES (sd) & $\mathrm{nr}$ & $15.4(3.0)$ & $11.6(4.3)$ & $11.7(4.1)$ \\
\hline Mean adult SES (sd) & $\mathrm{nr}$ & $13.3(3.5)$ & $11.7(4.6)$ & $12.5(4.4)$ \\
\hline Sample & $\begin{array}{l}\text { University } \\
\text { students, for } \\
\text { course credit }\end{array}$ & $\begin{array}{l}\text { University } \\
\text { students, for } \\
\text { course credit }\end{array}$ & $\begin{array}{l}\text { Online } \\
\text { participants, for } \\
\text { money }\end{array}$ & $\begin{array}{c}\text { Online } \\
\text { participants, for } \\
\text { money }\end{array}$ \\
\hline
\end{tabular}

Table 1. The characteristics of our study samples, compared to those in experiment 2 of Griskevicius et al (2011). sd = standard deviation, $n r=$ not reported. 


\begin{tabular}{|c|c|c|c|c|c|c|}
\hline \multirow[b]{2}{*}{ Primed perception } & \multicolumn{2}{|c|}{ Modified prime } & \multicolumn{2}{|c|}{ Original prime } & \multicolumn{2}{|c|}{ Prime comparison } \\
\hline & Mean & sd & Mean & sd & $\mathrm{t}$ & $\mathrm{p}$ \\
\hline Dangerous & 4.26 & 1.74 & 4.44 & 1.95 & -0.494 & 0.626 \\
\hline Unsafe & 4.17 & 1.83 & 4.61 & 1.75 & -1.146 & 0.264 \\
\hline Unpredictable & 3.87 & 1.87 & 4.74 & 1.71 & -2.237 & 0.036 \\
\hline Uncertain & 3.65 & 1.70 & 5.04 & 1.22 & -3.926 & 0.001 \\
\hline Arousal & 3.30 & 1.82 & 3.52 & 1.53 & -0.568 & 0.576 \\
\hline Convincing & 4.04 & 1.99 & - & - & - & - \\
\hline
\end{tabular}

360 Table 2. Means, standard deviations ( $s d$ ), and t-test results for the comparison between the

361 results from piloting our prime and those of prime piloting in the original study by 362 Griskevicius, Delton, et al. (2011). 


\begin{tabular}{|c|c|c|c|c|c|}
\hline Replication & Predictor & $\mathrm{F}$ & $\mathrm{p}$ & $\mathrm{B}$ & $\mathrm{SE}(\mathrm{B})$ \\
\hline \multirow[t]{4}{*}{ Replication 1} & \multicolumn{5}{|l|}{ Risk acceptance } \\
\hline & Condition & 1.78 & 0.19 & 0.43 & 0.32 \\
\hline & Child SES & 0.57 & 0.45 & -0.20 & 0.26 \\
\hline & Condition $*$ Child SES & 0.77 & $0.38\left(\mathrm{p}_{\mathrm{dir}}=0.24\right)$ & 0.29 & 0.33 \\
\hline \multicolumn{6}{|c|}{ Delay discounting } \\
\hline & Condition & 0.03 & 0.87 & 0.09 & 0.50 \\
\hline & Child SES & 0.24 & 0.62 & 0.20 & 0.40 \\
\hline & Condition $*$ Child SES & 0.10 & $0.76\left(\mathrm{p}_{\mathrm{dir}}=0.78\right)$ & -0.16 & 0.52 \\
\hline \multicolumn{6}{|c|}{ Risk acceptance } \\
\hline & Condition & 2.53 & 0.11 & -0.40 & 0.25 \\
\hline & Child SES & 3.40 & 0.07 & 0.30 & 0.16 \\
\hline & Condition $*$ Child SES & 0.03 & $0.87\left(\mathrm{p}_{\mathrm{dir}}=0.70\right)$ & -0.04 & 0.26 \\
\hline \multicolumn{6}{|c|}{ Delay discounting } \\
\hline & Condition & 2.03 & 0.16 & -0.54 & 0.38 \\
\hline & Child SES & 0.81 & 0.37 & -0.22 & 0.24 \\
\hline & Condition * Child SES & 1.82 & $0.18\left(\mathrm{p}_{\mathrm{dir}}=0.11\right)$ & 0.52 & 0.38 \\
\hline \multicolumn{6}{|c|}{ Risk acceptance } \\
\hline & Condition & 1.53 & 0.22 & 0.35 & 0.28 \\
\hline & Child SES & 5.26 & 0.02 & 0.45 & 0.19 \\
\hline & Condition $*$ Child SES & 0.59 & $0.44\left(\mathrm{p}_{\mathrm{dir}}=0.97\right)$ & -0.22 & 0.28 \\
\hline \multicolumn{6}{|c|}{ Delay discounting } \\
\hline & Condition & 0.10 & 0.75 & -0.13 & 0.42 \\
\hline & Child SES & 1.72 & 0.19 & 0.38 & 0.29 \\
\hline & Condition $*$ Child SES & 0.01 & $0.93\left(\mathrm{p}_{\mathrm{dir}}=0.58\right)$ & 0.04 & 0.43 \\
\hline
\end{tabular}

364 Table 3. Results from the main general linear models for the three replications. $D f=1,68$ represents p-value from a directed test as described by Griskevicius et al. (2011). 
367

\begin{tabular}{|c|cccc|}
\hline Risk acceptance & $\mathrm{F}$ & $\mathrm{p}$ & $\mathrm{B}$ & $\mathrm{SE}(\mathrm{B})$ \\
\hline Unsafe & 5.61 & 0.02 & -0.37 & 0.16 \\
Child SES & 0.85 & 0.36 & 0.75 & 0.82 \\
Unsafe * Child SES & 0.43 & 0.51 & -0.10 & 0.15 \\
\hline Delay discounting & $\mathrm{F}$ & $\mathrm{p}$ & $\mathrm{B}$ & $\mathrm{SE}(\mathrm{B})$ \\
\hline Dangerous & 4.73 & 0.03 & 0.45 & 0.21 \\
Child SES & 0.20 & 0.66 & 0.41 & 0.92 \\
Dangerous * Child SES & 0.03 & 0.86 & -0.03 & 0.17 \\
\hline
\end{tabular}

368 Table 4. Results of the model examining interaction effects for primed danger perceptions 369 and child SES scores on delay discounting in replication 3. $D f=1,82$. "Unsafe" refers to 370 participants responses to the question "To what extent did the story make you think the world 371 will become unsafe?", and "Dangerous" refers to participants responses to the question "To 372 what extent did the story make you think the world will become a more dangerous place?" 
373

(a)

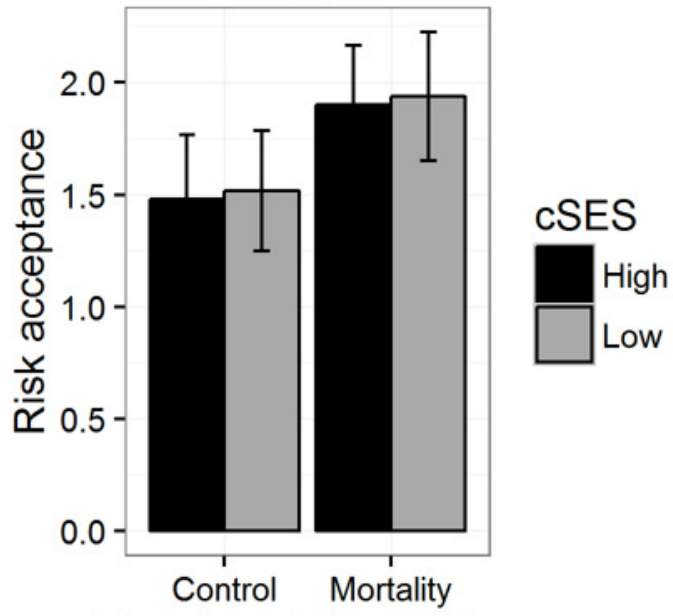

Experimental condition (b)

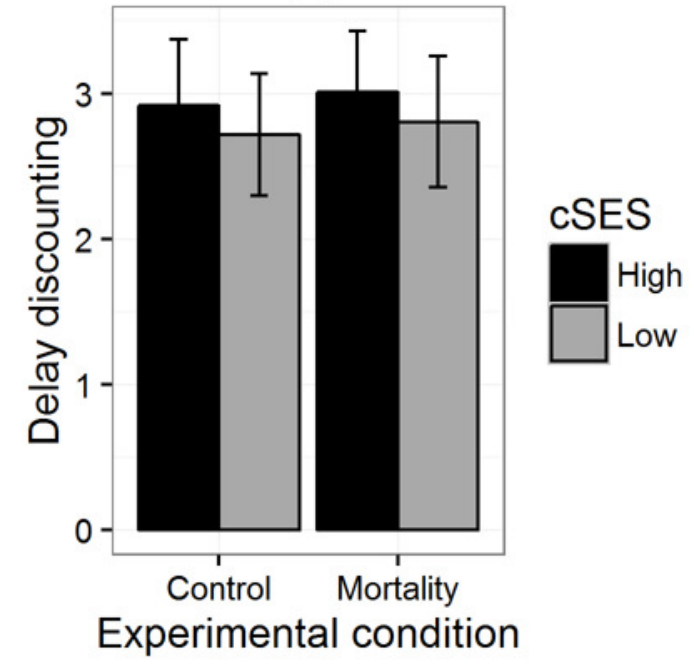

Figure 1. Risk acceptance (a) and delay discounting (b) by priming condition for participants of high and low childhood SES in replication 1. Error bars represent one standard error. 
377

(a)

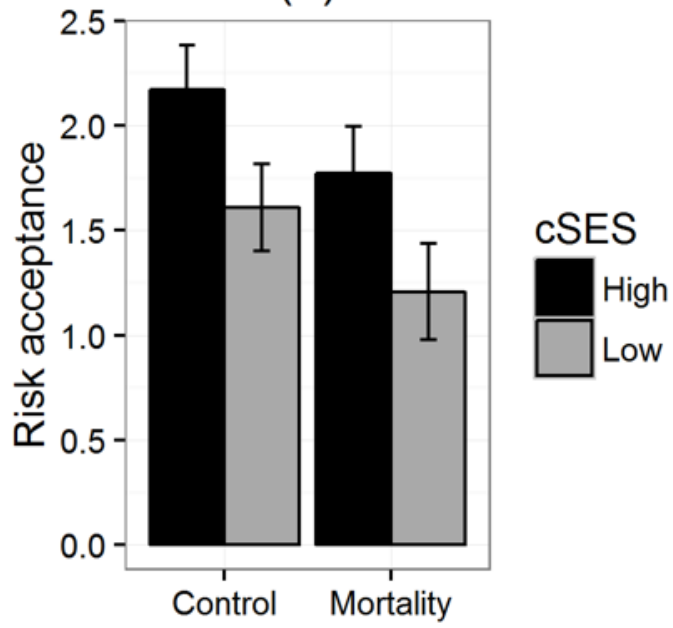

Experimental condition (b)

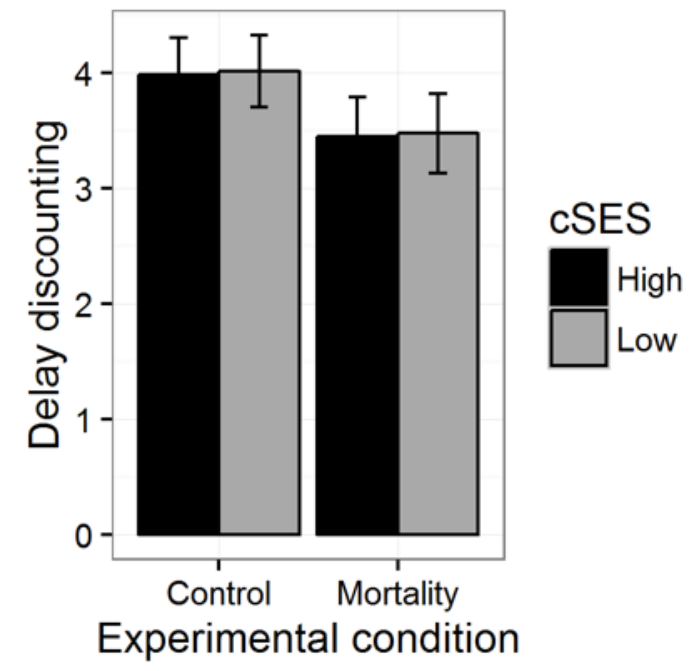

Figure 2. Risk acceptance (a) and delay discounting (b) by priming condition for participants of high and low childhood SES in replication 2. Error bars represent one standard error. 
381

(a)

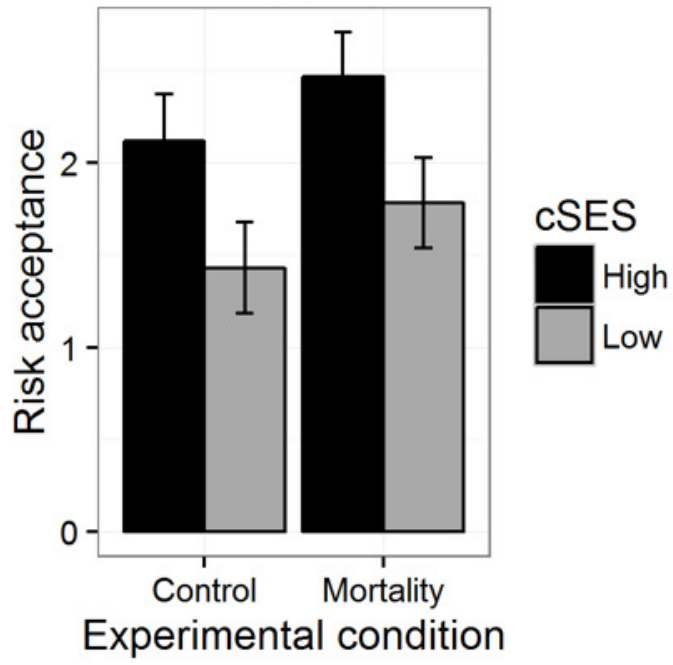

(b)

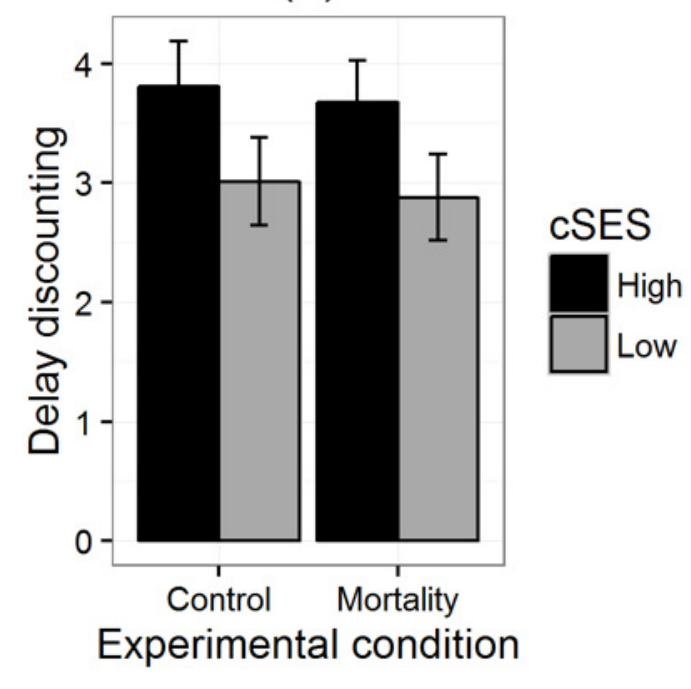

Figure 3. Risk acceptance (a) and delay discounting (b) by priming condition for participants of high and low childhood SES in replication 3. Error bars represent one standard error. 
(a) Main effect of condition, risk acceptance

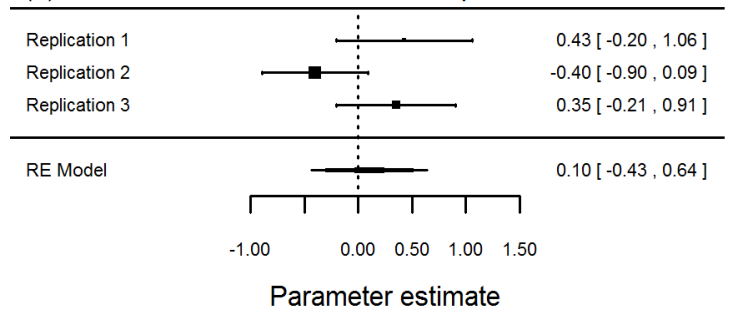

(c) Main effect of childhood SES, risk acceptance

\begin{tabular}{|c|c|c|c|c|c|c|}
\hline \multirow{3}{*}{$\begin{array}{l}\text { Replication } 1 \\
\text { Replication } 2 \\
\text { Replication } 3\end{array}$} & & & $\vdots$ & & & $-0.20[-0.70,0.31]$ \\
\hline & & & $\vdots$ & _ & & $0.30[-0.02,0.61]$ \\
\hline & & & & & & $0.45[0.06,0.83]$ \\
\hline \multirow[t]{3}{*}{ RE Model } & & & $\div$ & & & \multirow[t]{2}{*}{$0.22[-0.10,0.55]$} \\
\hline & $\Gamma$ & $T$ & $i$ & $T$ & \urcorner & \\
\hline & -1.00 & -0.50 & 0.00 & 0.50 & 1.00 & \\
\hline
\end{tabular}

(e) Interaction, risk acceptance

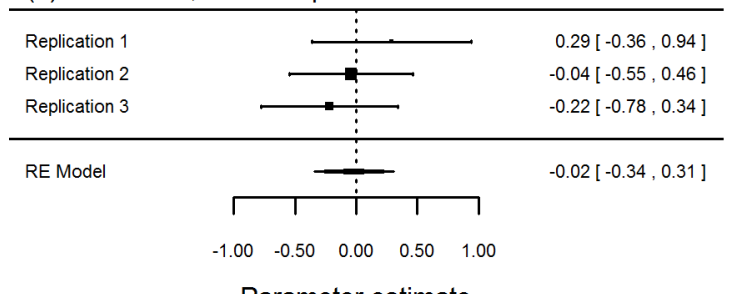

(b) Main effect of condition, delay discounting

\begin{tabular}{|c|c|c|c|c|c|}
\hline Replication 1 & & & & \multicolumn{2}{|r|}{$0.09[-0.91,1.08]$} \\
\hline Replication 2 & & $\rightarrow-$ & & & $-0.54[-1.28,0.20]$ \\
\hline Replication 3 & & & & & $-0.13[-0.97,0.70]$ \\
\hline \multirow[t]{3}{*}{ RE Model } & & & & & $-0.25[-0.74,0.23]$ \\
\hline & $\Gamma$ & $T$ & $T$ & 7 & \\
\hline & -1.50 & -0.50 & 0.50 & 1.50 & \\
\hline
\end{tabular}

(d) Main effect of childhood SES, delay discounting

\begin{tabular}{|c|c|c|c|c|c|c|}
\hline \multicolumn{2}{|l|}{ Replication 1} & & & & 一 & $0.20[-0.60,1.00]$ \\
\hline Replication 2 & & & $1:$ & & & $-0.22[-0.69,0.26]$ \\
\hline Replication 3 & & & $\div$ & - & 一 & $0.38[-0.19,0.95]$ \\
\hline \multirow[t]{3}{*}{ RE Model } & & & 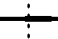 & & & $0.08[-0.33,0.50]$ \\
\hline & 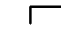 & T & $T$ & T & T & 1 \\
\hline & -1.00 & -0.50 & 0.00 & 0.50 & 1.00 & 1.50 \\
\hline
\end{tabular}

(f) Interaction, delay discounting

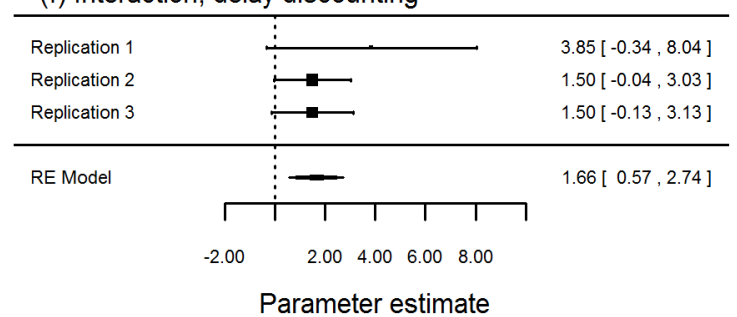

Figure 4. Forest plots from meta-analyses across our three experiments, showing the main effects of mortality priming condition (panels $a$, b); the main effects of childhood SES (panels $c, d$ ), and interaction between mortality-priming condition and childhood SES (panels e, f). 